\title{
Space Weather Impact on Magnetosphere: New Helium Radiation Belt Storm Time Formation
}

\author{
D. Boscher ${ }^{1}$, S. Bourdarie ${ }^{1}$, A.A. Gusev², U. Jayanthi², I.M. Martin ${ }^{3}$, \\ G.I. Pugacheva ${ }^{2}$, and W.N. Spjeldvik ${ }^{4}$ \\ ${ }^{1}$ ONERA/DESP - 2, av. E. Belin, BP 4025, 31055 Toulouse Cedex4, France \\ ${ }^{2}$ Instituto Nacional de Pesquisas Espaciais/INPE, 12201-970, São José dos Campos, SP, Brazil \\ ${ }^{3}$ University of Campinas, Instituto de Física/DRCC, 13083-970, Campinas, SP, Brazil \\ ${ }^{4}$ Department of Physics, Weber State University, Ogden, Utah 84408-2508
}

Received on 28 November, 2001

\begin{abstract}
The consequences of the March 1991 storm on the Earth's radiation environment are now well known through many in-situ magnetospheric observations. Measurement from the CRRES spacecraft show the sudden formation of a new Helium ion structure deep within the magnetosphere. In that event, two additional new radiation belts were created, one containing large fluxes of $10 \mathrm{MeV}$ electrons, and the other forming a structure containing $30 \mathrm{MeV}$ protons. These sudden space weather phenomena appear to be due the conjunction of the two occurrences: (1) a solar energetic particle (SEP) event arriving in the vicinity of the Earth, and (2) a very intense magnetic storm within the Earth's magnetosphere. Attempts to reproduce the appearance of storm time helium belt by computational modeling are made using a three-dimensional charged particle code (Salammbo code) incorporating Helium ion charge exchange and post-event classical diffusion theory. We have extended the capabilities of the code to Helium ions, and we show that these physical phenomena can also lead to a build-up of a new Helium belt in the $30 \mathrm{MeV}$ range. Detailed modeling predictions of the location of this belt and computed flux levels will be presented and discussed in the context of space weather phenomena in the Earth's magnetosphere.
\end{abstract}

\section{Introduction}

The Earth's radiation belt region is a dynamic entity that readily responds to changes in the solar energetic particle environment. With the launch of many Earth orbiting spacecraft with telescopes, one has the ability to measure and monitor the radiation belt region in considerable details. Advances in numerical modeling of this radiation environment has brought forth simulation and prediction capabilities of great utility for eventual space weather forecast. In the early part of the space era, the radiation belt modeling was carried out by both experimental and theoretical means [1-5] to understand the sources and losses of the great belt fluxes and the Space Weather Program ushered in a dynamical modeling of radiation belt. Two-dimensional modeling of the radiation belt flux enhancement for energetic protons have been carried out with time variable diffusive transport coefficients and time variable (but fixed location) boundary conditions [6]. Variations in the geomagnetic rigidity cut-off locations with particle energy and geomagnetic conditions, as well as the solar energetic flux (SEP) intensity levels have not been attempted until now. We attempt here more comprehensive modeling.

Modern observations have shown that there can be large variations in the geomagnetically confined radiation, even deep within the trapping region [7-9]. The physical consequences of the March 1991 magnetic storm in the Earth's radiation environment are now reasonably well known through many in-situ magnetospheric observations. Measurement made with the CRRES spacecraft show a sudden formation of a new geomagnetically confined energetic radiation structure consisting of several different charged particle species, including a new Helium ion radiation belt, deep within the Earth's magnetosphere, and in that event, several additional new radiation belts were rather suddenly created, one containing large fluxes of $10 \mathrm{MeV}$ electrons, another containing $30 \mathrm{MeV}$ protons.

These abrupt space weather storm-phenomena appear to be due to the conjunction of the two occurrences: (1) a solar energetic particle (SEP) event arriving in the vicinity of the Earth, and (2) a very intense magnetic storm within the Earth's magnetosphere itself. In the Vacaresse et al. time dependent proton 
model ${ }^{10}$, it was shown that with increased geomagnetic turbulence and availability of enhanced particle fluxes impulsively formed proton radiation belts can be modeled with location and intensity in good agreement with measurements made by the CRRES satellite. Further tests of this modeling must include other particle species, and we here address the creation of an inner radiation zone Helium ion belt.

\section{Processes included in model- ing}

The existing Salammbo code for 3-dimensional calculations of radiation belt proton distributions [11] was extended to Helium ions by the inclusion of both the ionic states and the coupling between them [12-14]. In short, Helium ions can appear in two different charge states $(\mathrm{He} 1+$ and $\mathrm{He} 2+)$ with charge exchange coupling between the two species induced by collisional interactions in the exospheric thermal neutral hydrogen environment. The natural radial transport of Helium is thus occurring simultaneously with the collisional processes. The diffusion equations governing these two charge states of Helium are coupled. In phase space spanned with the three adiabatic invariants, the two coupled transport equations are written as:

$$
\begin{gathered}
\frac{\partial f_{++}}{\partial t}=L^{2} \frac{\partial}{\partial L}\left(\frac{D_{L L_{++}}}{L^{2}} \frac{\partial f_{++}}{\partial L}\right)+\frac{\partial}{\partial M}\left(\left(\frac{\partial M}{\partial t}\right)_{++} f_{++}\right)+ \\
\frac{\partial}{\partial J}\left(\left(\frac{\partial J}{\partial t}\right)_{++} f_{++}\right)+\frac{f_{+}}{\tau_{+/++}}-\frac{f_{++}}{\tau_{++/+}}, \\
\frac{\partial f_{+}}{\partial t}=L^{2} \frac{\partial}{\partial L}\left(\frac{D_{L L_{+}}}{L^{2}} \frac{\partial f_{+}}{\partial L}\right)+\frac{\partial}{\partial M}\left(\left(\frac{\partial M}{\partial t}\right)_{+} f_{+}\right)+ \\
\frac{\partial}{\partial J}\left(\left(\frac{\partial J}{\partial t}\right)_{+} f_{+}\right)-\frac{f_{+}}{\tau_{0 /+}}-\frac{f_{+}}{\tau_{+/++}}+\frac{f_{++}}{\tau_{++/+}}
\end{gathered}
$$

where $f_{+}$and $f_{++}$are respectively the distribution functions (phase space densities) corresponding to the He1+ and $\mathrm{He} 2+$ populations, M, J, and L are respectively the relativistic magnetic moment, the second adiabatic invariant and the McIlwain L-shell parameter. The $\mathrm{D}_{L L}$ coefficients are the radial diffusion coefficients, which can be different for the two ionic states of the same elemental species due to the charge dependent drift velocities.

Following the earlier success of the proton code in 3-D time dependent simulations [10], the Helium ion radial diffusion coefficients were assumed to be magnetic activity dependent through the magnetic activity index, $\mathrm{Kp}$. We assumed the $\mathrm{D}_{L L}$-values to be proportional to $+\exp (0.74 \bullet \mathrm{Kp})$, following the dependence deduced from measurements at synchronous orbit [15].
The coefficients, $\mathrm{dM} / \mathrm{dt}$ and $\mathrm{d} \mathrm{J} / \mathrm{dt}$, are derived from the effective Coulomb energy degradation rates, $\mathrm{dE} / \mathrm{dt}$, due to non-charge-exchange interactions with the neutral of the exosphere. Finally, the coupling coefficients $\tau_{o /+}, \tau_{+/++}$and $\tau_{++/+}$are relative to the charge exchange reactions. The charge exchange cross section were derived from laboratory measurements [13].

In the present Helium ion simulation, no internal radiation belt sources were considered, and the Helium ion radiation belts are thought to be filled by sources exterior, either from the magnetotail (or direct entry from the magnetosheath) or from the Earth's ionosphere via high latitude processes. Globally, the process flow chart of the trapped Helium population is exhibited in Fig. 1. 


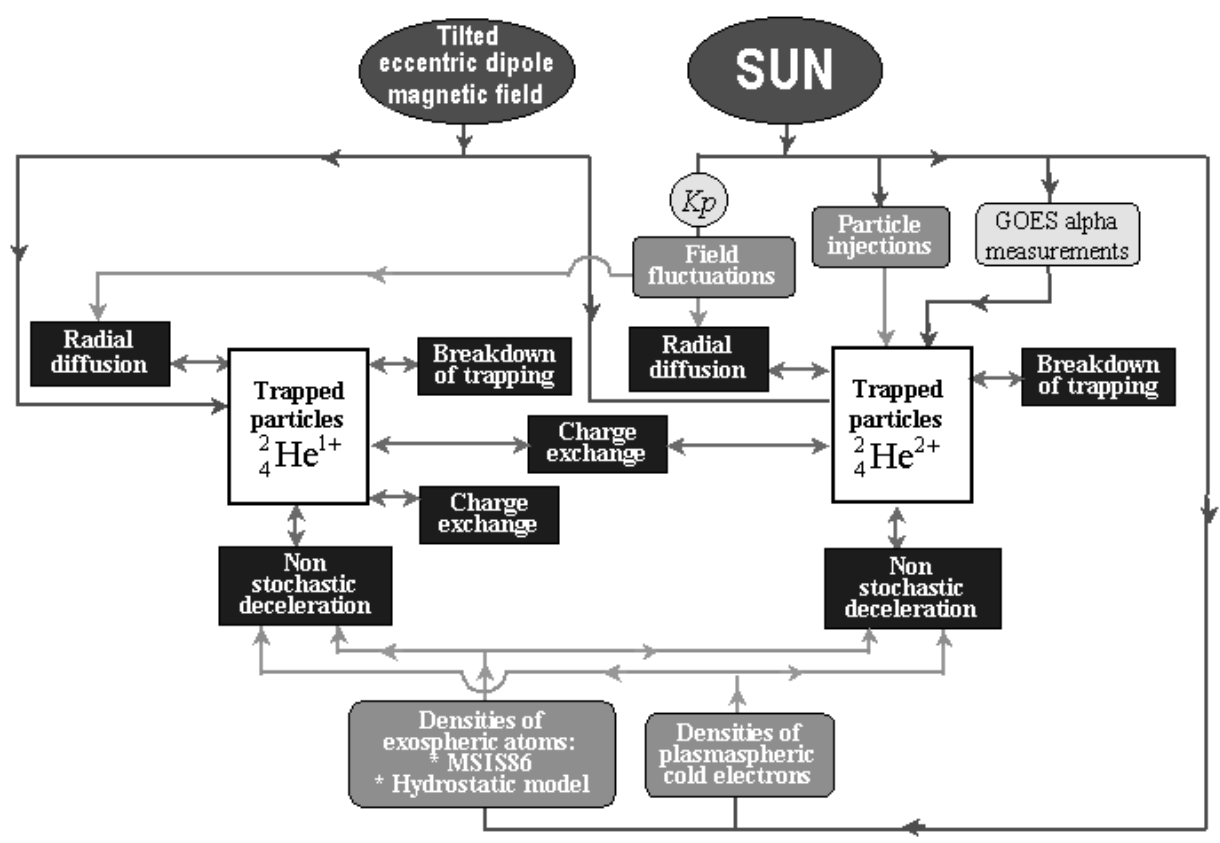

Figure 1. Flow chart of the Helim trapped radiation model.

\section{Boundary conditions}

The trapping region is limited to low altitudes by the high atmosphere, with nominal $100 \mathrm{~km}$ height. This limits the access of Helium ions to low L-shells, and it also provides an effective boundary in pitch angle space for higher L-shells. As in the earlier work, at high altitudes, the nominal outer zone boundary was taken as $\mathrm{L}$ $=7$ for very quiet conditions, but we found it necessary to modify this traditional boundary when significant events take place so that the effective outer boundary adjusts according to variations in the geomagnetic field topology. In this regard it was desirable to obtain Helium ion flux simulations of very energetic ions (up to $300 \mathrm{MeV}$ ), and it was necessary to include the magnetic cut-off of the Helium ions. This cut-off is related to the large Larmor radius of Helium ions compared to the scale lengths of the gradients in the magnetic field.

As ions approach the Earth magnetic field from interplanetary space, they deviate from their preencounter trajectories. The minimum radial location they can penetrate into the terrestrial magnetosphere depends on the particle magnetic rigidity, a property dependent on Helium ion energy and charge state as well as the geomagnetic field encountered. We here assume that the cut-off energy $\mathrm{E}_{\text {cutoff }}$ on a geomagnetic drift shell location defined by the L-parameter does not depend on the Helium ion pitch angle, and can be expressed as: $\mathrm{E}_{\text {cutoff }}=\exp \left(\mathrm{aL}^{2}+\mathrm{bL}+\mathrm{c}\right)$ [in $\mathrm{MeV}$ ], with the coefficients $\mathrm{a}, \mathrm{b}$ and $\mathrm{c}$ dependent on the spe- cific ion species (Helium ion in our case) deduced from the empirical information shown in Fig. 2.

\section{Radiation belt Helium model: geomagnetic cut-off in a dipole field}

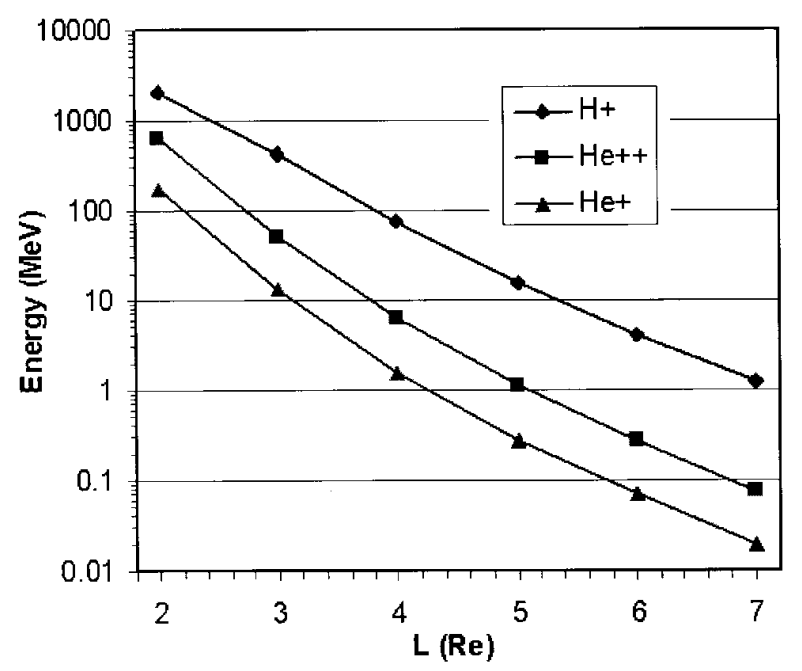

Figure 2. Empirically modeled cut-off energies versus the geomagnetic L-shell parameter for the two charge states of Helium. Comparison is also made with the same conditions for protons.

At very high energies, because of their high magnetic rigidity, charged particles from the interplanetary medium have direct access to substantial portions 
of the internal magnetosphere, including the radiation belt region. To establish the realistic magnetic cut-offs we used measurements of alpha particles $(\mathrm{He} 2+)$ fluxes from the NOAA/GOES-6 geosynchronous satellite as boundary conditions, and we assumed that the same fluxes would be present at lower L-shell down to the rigidity cut-off location. To establish a full spectrum, we extended the empirical GOES-6 Helium spectra towards lower energies. Boundary conditions were fixed to zero during the whole period for He1+, assuming that the sun predominantly supplies $\mathrm{He} 2+$ ions. In analogy with the atomic Oxygen ion work of Spjeldvik and Fritz [13], this permits a parameterized understanding of the effect of the ionic charge exchange.

\section{Diffusive transport modeling}

We have built a numerical model to solve the coupled governing transport equations for energetic Helium ions with two built-in algorithms: (1) A direct iterative equilibrium state seeker with test routines to insure that equilibrium was indeed attained; and (2) A forward time-stepper to simulate time progression during events of interest, either starting from an arbitrary initial state or from the computed equilibrium configuration. This model has been used to gain detailed knowledge of the dynamical evolution of the Helium radiation belt structure.

To gain insight into the coupled processes in the 3$\mathrm{D}$ radiation belt confinement region, we wanted to see clearly the time variable effects of different processes (ionic charge exchange, Coulomb energy degradation, cross-L transport, and the effect of a Solar Energetic Particle Event). We here report results from some of these simulations where we used as initial conditions an otherwise empty magnetosphere and let it be filled by cross-field transport from an outer edge source specified as a boundary condition.

With this starting point attained we proceeded to model the transport of particles during the great March 1991 storm. Numerical computations were made simulating the structure of the Helium fluxes for the period between 23 March and 2 April, 1991. To insure realism we used the corresponding $\mathrm{Kp}$ values and GOES-6 alpha particle measurements (Fig. 3) to mimic the geomagnetic response to the solar-induced disturbances. This we did with both $\mathrm{D}_{L L}$-values $\mathrm{Kp}$-dependent and the geomagnetic cut-off energy (and thus the corresponding cut-off location for a given energy). It should be noticed that the interesting Solar Energetic Particle Event begin on 23 March at round 10:00 am while the storm, seen with the Kp index, begins on 24 March at around 3:00 am, nearly one day later.

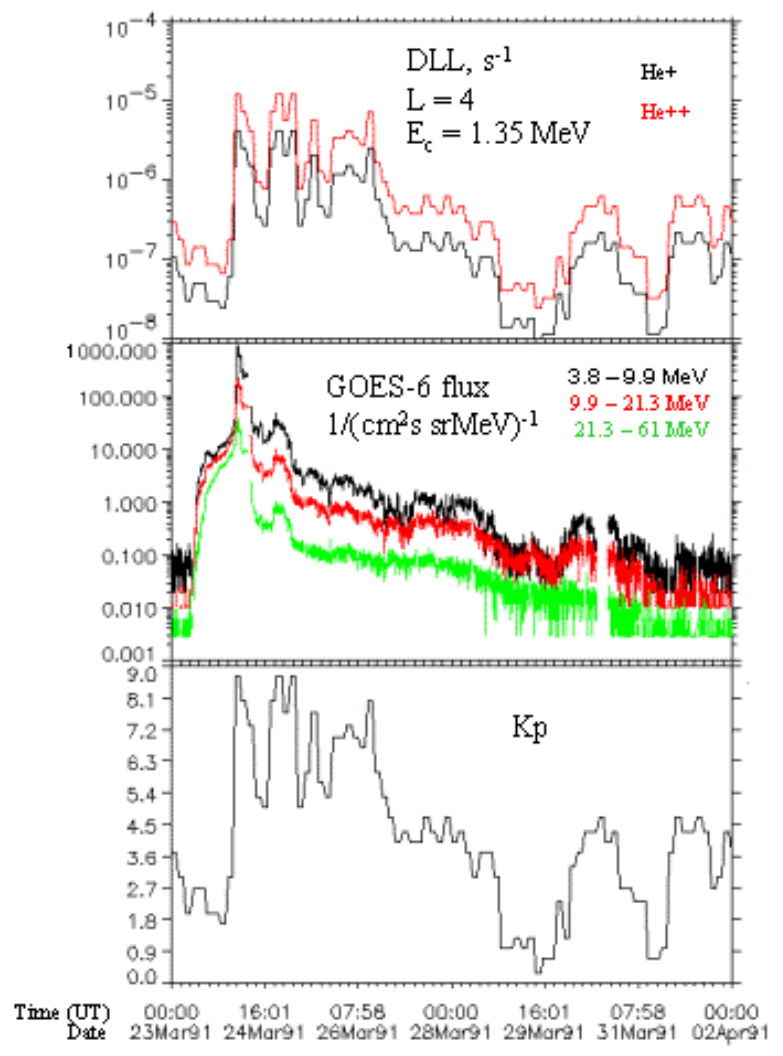

Figure 3. Time dependent input parameters applied for the numerical model run. From bottom to top: (1) the geomagnetic index $\mathrm{Kp}$; (2) alpha particle measurements with the GOES-6 satellite in three different energy channels; (3) modeled $\mathrm{Kp}$-dependent radial diffusion coefficients versus time at $\mathrm{L}=4$ for $1.35 \mathrm{MeV}$ He particles in either charge state.

\section{Results of the numerical sim- ulations}

The results of the numerical simulations are illustrated in Fig. 4 which depicts the Helium ion flux distributions for $\mathrm{He} 1+$ and $\mathrm{He} 2+$ presented as omni-directional fluxes versus time (abscissa axis) and geomagnetic Lshell parameter (ordinate axis). Regarding the He2+ ions, we can understand this result in terms of lower energy, particles continuously injected from the geomagnetic tail, and so made available to the outer edge of the stable confinement region at a nominal location of $\mathrm{L}=7$. In the present model, these Helium ions are transported to lower L shells by time dependent radial diffusion.

Let us first consider the range of moderate Helium ion energies, in the tens and hundred of $\mathrm{keV}$ range. When the availability of Helium ions in the outer zone is substantial and the Kp index is high (i.e., the Earth's magnetosphere is strongly disturbed), radial transport is very efficient, particularly in the outer parts of the 
trapping region, so that the He flux seen at $\mathrm{L}=4$ growths rapidly. When $\mathrm{Kp}$ decreases, this transport process slows down, and the in-situ charge exchange from $\mathrm{He} 2+$ to He1+ becomes a very efficient loss for the originally injected $\mathrm{He} 2+$ particles. Thus the $\mathrm{He} 2+$ flux decreases rapidly while the flux of He1+ ions increases. Further charge exchange from He1+ to neutral energetic Helium acts to untrap the particles, and these are then lost almost instantaneously from the radiation belts. Some energetic Helium disappear to outer space while others precipitate into the atmosphere where secondary collisional reactions take place.
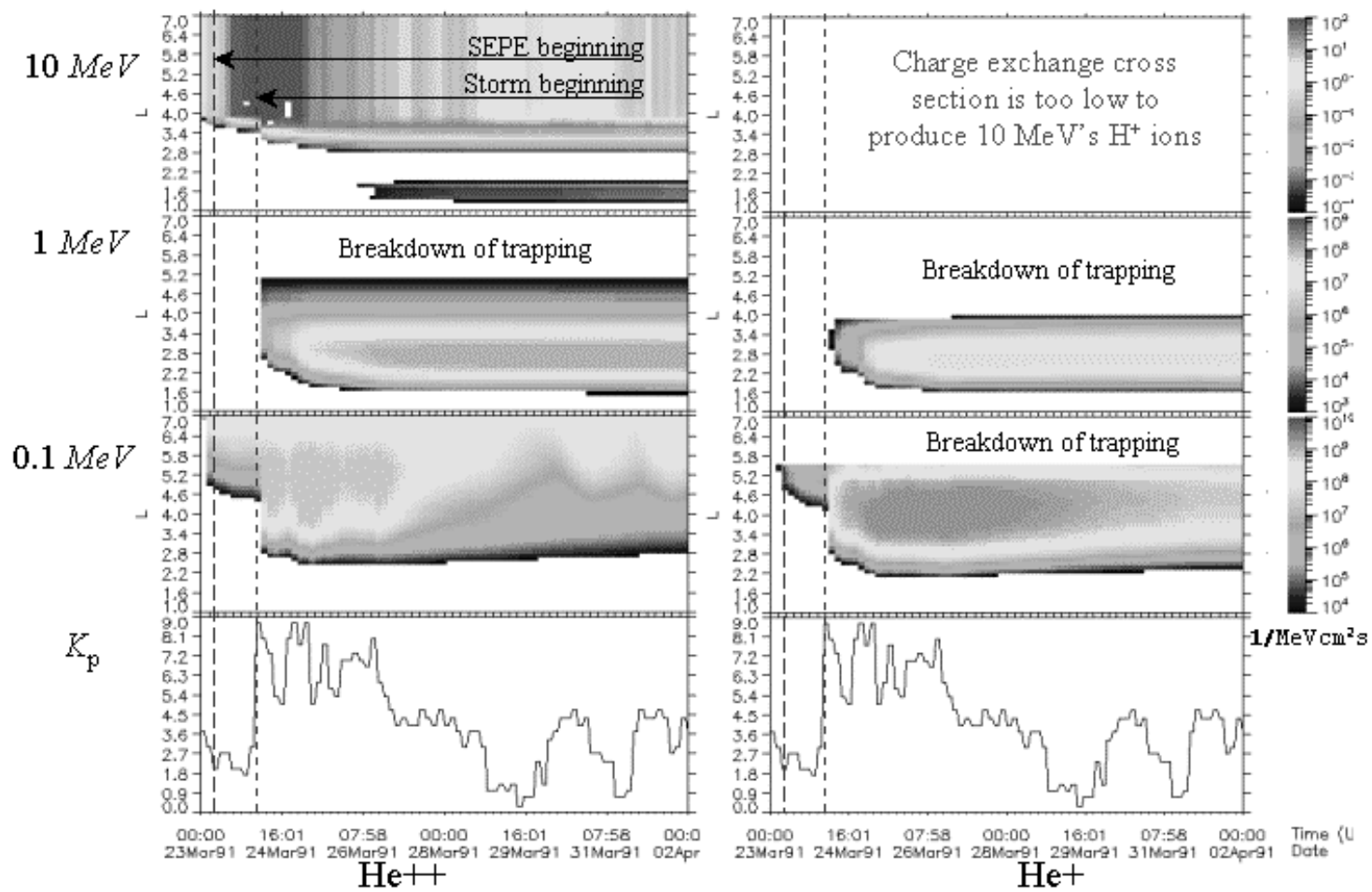

Figure 4. Results of the numerical simulations for both species in terms of equatorial omnidirectional flux in a L (ordinate) versus time (abscissa) plot for three representative energies (100 keV, $1 \mathrm{MeV}$ and $10 \mathrm{MeV}$ ).

The effective transport time constant for the $1 \mathrm{MeV}$ Helium ions population is longer, so the Helium ion flux grows more slowly at $\mathrm{L}=4$. These fluxes continue to increase as long as the storm injection effects are present, and it becomes almost constant afterwards due the to lower rates of charge exchange losses than compared to the lower energy Helium ions. These particles can be trapped for long periods of time. The white colored portion of the plot for $\mathrm{L}$ greater than 5 corresponds to particles crossing the magnetosphere, because the geomagnetic rigidity cut-off energy corresponds to $1 \mathrm{MeV}$ at $\mathrm{L}=5$ (Fig. 2). Beyond this cut-off, the flux is below the minimum of the scale, and is not seen in the figure. At higher energies (say, $10 \mathrm{MeV}$ ), the geomagnetic rigidity cut-off location is closer to the Earth (around $\mathrm{L}=3.8$ ).

Particles from the Solar Energetic Particle Event are seen between this cut-off L-shell location and the boundary, and the flux is taken to be constant between these two L-values. Thus the time dependency of this flux in this region follows the GOES-6 Helium flux mea- surements, shown in Fig. 3. During the geomagnetic storm, these Helium ions cross into the magnetosphere and have free access to the trapping region due to the time dependent rigidity cut-off location, the time dependence of this value being caused by the changing geomagnetic field topology during the event. The combined effect of (1) enhanced Helium ion flux availability at the edge of the trapping region, (2) enhanced and time variable cross-L transport, and (3) time variable geomagnetic rigidity cut-off location cause the formation of a new (and temporary) Helium ion radiation belt at lower L-values (here around $\mathrm{L}=3.6$ at $1 \mathrm{MeV}$ ), constituting a new $\mathrm{He} 2+$ belt.

In our model parameters, singly charged ions $(\mathrm{He} 1+)$ are formed only by charge exchange from $\mathrm{He} 2+$. Because the charge exchange process is far more efficient at lower Helium ion energy, the flux of this charge species $(\mathrm{He} 1+)$ is greater at $100 \mathrm{keV}$ than at $1 \mathrm{MeV}$. Computationally, they appear quasi instantaneously after the alpha particle fluxes have entered. One should also notice that the rigidity cut-offs for $\mathrm{He} 1+$ ions are 
lower than the cut-offs for alpha particles, and this implies that it is practically impossible to see $\mathrm{He} 1+$ particles at higher L-shells beyond $\mathrm{L}=5.8$ for $100 \mathrm{keV}$. According to this simulation, $1 \mathrm{MeV} \mathrm{He}+$ particles are predominantly produced near $\mathrm{L}=2.8$.

The distributions (omnidirectional flux versus L) for the two species are plotted at $1 \mathrm{MeV}$ at the temporal end of the simulation, and this is depicted in Fig. 5 . The radial profiles of $\mathrm{He} 1+$ and $\mathrm{He} 2+$ are different, this being essentially an effect of the charge dependent rigidity cut-off of the two Helium ion charge states.
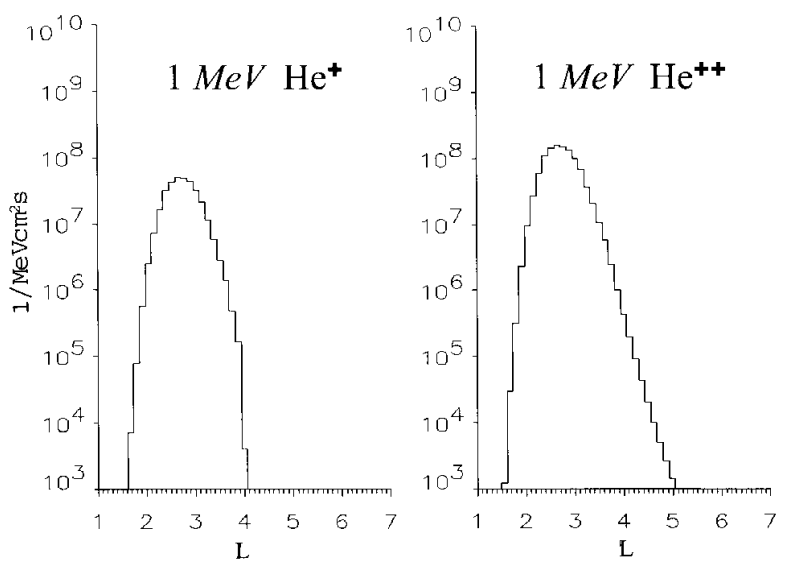

Figure 5. Distributions (equatorial omnidirectional differential flux versus L-shell) of $\mathrm{He} 1+$ and $\mathrm{He} 2+$ at $1 \mathrm{MeV}$ at the end of the simulation run.

The Helium ion flux distributions at different periods in the simulation interval are plotted in Fig. 6 for the $10 \mathrm{MeV}$ particles. The geomagnetic rigidity cut-off for the particles is clearly seen in this figure as the flux then is constant in $\mathrm{L}$ (vertical stripes in this display) between the energy-dependent rigidity cut-off location and our nominal boundary at $\mathrm{L}=7$. This flux of course varies with time as the availability of outer edge fluxes vary (as seen with GOES-6), and the initial value at this location is re-established when the Solar Energetic Particle Event is over. At the end of the numerical simulation, the result is that a new "diffusively injected" radiation belt was created by the conjunction between the enhanced flux availability event and the intense geomagnetic storm in the magnetosphere. The maximum fluxes of this additional radiation belt is modeled to be located at $\mathrm{L}=3.6$ and the omni-directional flux there reaches $400 \mathrm{MeV}^{-1} \mathrm{~cm}^{-2} \mathrm{~s}^{-1}$.

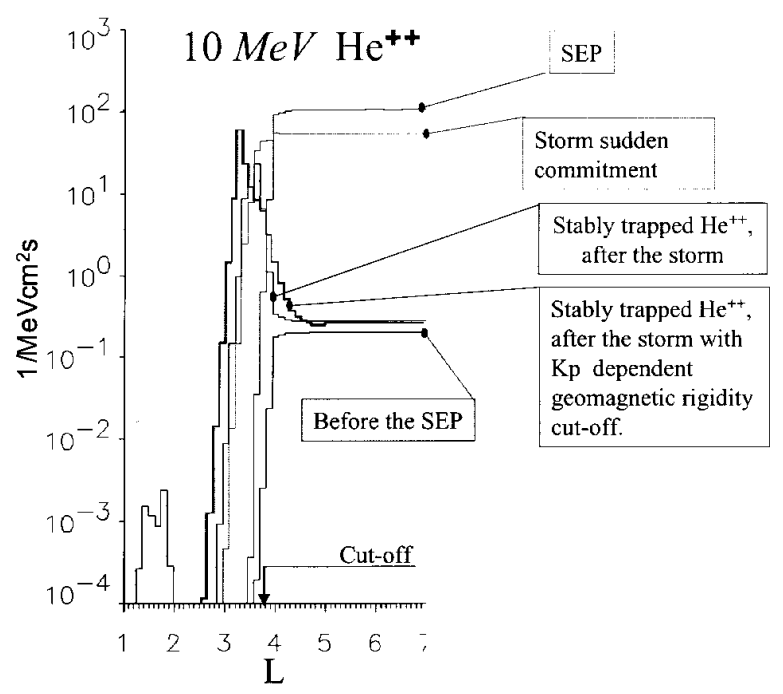

Figure 6. Distribution of equatorial omni-directional He2+ particles at different instants during the simulated event period.

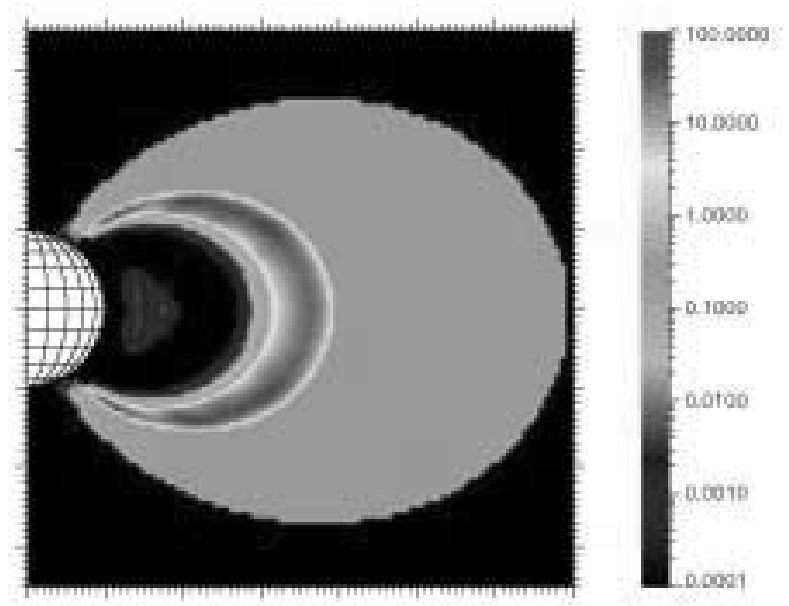

Figure 7. Omnidirectional differential flux (in units of: ions MeV-1 cm-2 s-1) of $1 \mathrm{MeV} \mathrm{He} 1+$ and $\mathrm{He} 2+$ ions at the end of the simulation time span. It can be seen that the alpha particle $(\mathrm{He} 2+)$ radiation belt generated in this particular event also extends down to low altitudes along the same L shell.

This time-dependent and charge state coupled physical model is developed not only for equatorial particles, but also outside the geomagnetic equator and includes the full range of equatorial pitch angles for Helium ions outside the atmospheric bounce loss cone. The entire radiation belt created at $10 \mathrm{MeV}$ at the end of the simulation can be seen in Fig. 7. This model thus provides a quantitative explanation for the rapid formation of new temporal radiation belts within the classical trapping region. 


\section{Discussion and Conclusion}

With this simple model that employs a combination of classical diffusion theory concepts (time variable radial transport, ionic charge exchange and Coulomb collisional energy degradation) coupled with the idea of time variable geomagnetic rigidity cut-offs due to geomagnetic topology variations, it was possible to create an alpha particle radiation belt at $\mathrm{L}=3.6$ for 10 $\mathrm{MeV}$ and corresponding structures at different energies. The necessary conditions are a Solar Energetic Particle Event, which brings these particles near the outer radiation zone trapping boundary (effectively the geomagnetic rigidity cut-off location for a given energy) and an intense geomagnetic storm, which transport these particles in the trapping region down to lower L shells.

The final L-shell of the effective insertion of these particles is a combination of the cut-off $(\mathrm{L}=3.8$ at 10 $\mathrm{MeV}$ ) and the range of L-shells they can diffusively cross once within the rigidity cut-off L-value. These co-occurrences depend on the intensity as well as the duration of the storm. In fact, the L-shell range of the diffusive process is here rather small (around delta $\mathrm{L}=$ 0.2 , so the main "injector-mechanism" is the temporal variation of the geomagnetic rigidity cut-off location in the inner magnetosphere. Of course, in cases where the duration and intensity of the geomagnetic storm are large, effective L-shell crossing by diffusion can also be important and an additional mechanism.

To emphasize the importance of the geomagnetic topology variations during geomagnetic storms, we note that the geomagnetic rigidity cut-off is certainly substantially modified during the progression of an intense magnetic storm. In this paper we have provided the first quantitative attempt at modeling using a Kp dependent cut-off condition with a $\mathrm{E}_{\text {cutoff }}$-formula (and thus equivalently a $\mathrm{L}_{\text {cutoff }}$-location for a given energy) derived from the empirical cut-off versus $\mathrm{L}$ relationship $\left.\mathrm{E}_{\text {cutof } f}=\exp \left(\mathrm{a} \mathrm{L}^{2}+\mathrm{bL}+\mathrm{c}\right)\right)$. In fact, in the present numerical simulations we used only a normalizing factor for $\mathrm{L}$ in this equation, this factor being equal to $(3+4 \exp (-\mathrm{Kp} / 3.06)) / 3.78^{[10]}$, so that it is equal to unity for $\mathrm{Kp}=5$. This means that energetic Helium ions go inside the limit seen in Fig. 2 for even higher Kp values, and lie outside for lower $\mathrm{Kp}$ values. With this formula, the geomagnetic rigidity cut-off for $10 \mathrm{MeV}$ alpha particles at $\mathrm{Kp}=9$ comes down as far as to $\mathrm{L}$ = 3.2. The distribution of the $10 \mathrm{MeV}$ alpha particles with this run is shown in Fig. 8 at the end of the simulation.

It can be seen that the maximum omni-directional flux of the newly formed belt in this second simulation is found at $\mathrm{L}=3.1$, and is a little bit higher in flux intensity, around 800 ions $\mathrm{MeV}^{-1} \mathrm{~cm}^{-2} \mathrm{~s}^{-1}$. It then appears that the maximum Helium ion flux intensity level depends not only on the outer zone incident spectrum of the event, but also on the location of the geomag- netic rigidity cut-off during the storm. The characteristic width of the new Helium ion radiation belt also significantly depends on the effective radial diffusion coefficients and on the temporal displacement history of the magnetic rigidity cut-offs during the storm.

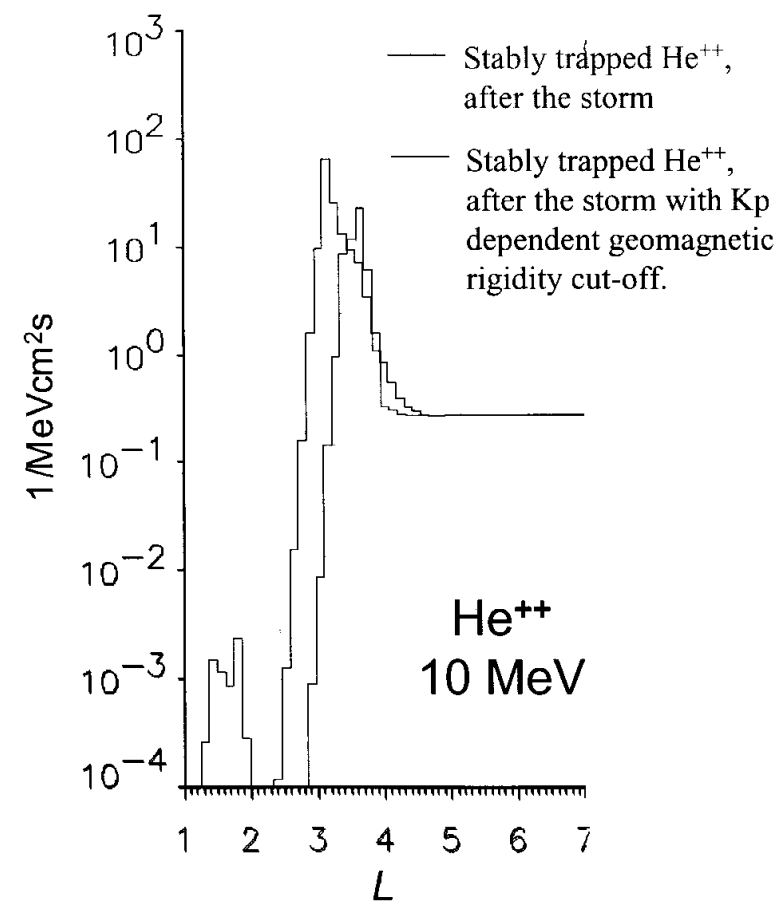

Figure 8. Distribution of equatorial $\mathrm{He} 2+$ ions at the end of the simulation, which uses a Kp dependent geomagnetic rigidity cut-off.

With the present numerical model, the impulsively formed radiation belt is created over all the energies where Helium ion flux during the Solar Energetic Particle Event is greater than the background due to cosmic rays. Depending on the cut-off locations and the intensity of a particular storm, this new Helium ion radiation belt structure can be effectively created well inside the classical trapping region. This may well at times come to broaden in geomagnetic invariant latitudes and may possibly interfere with man-made structures and operations in space, including the International Space Station. Under worst case scenarios it is possible that newly created radiation belts of electrons, protons, Helium ions and even heavier ion species may endanger the health or life of astronauts, especially during Extra Vehicular Activities.

\section{Acknowledgments}

Figures 3, 4, and 7 were made using the PAPCO data analysis package supported by MPAE Lindau through the Max-Planck Gesellschaft, DARA,grant 50 OC 95010, and as part of the HYDRA NASA funding under grant number NAG 52231. 


\section{References}

[1] J. Zinn, H. Hoerline, and A. G. Petchek, The Motion of Bomb Debris Following the Starfish Test, in Radiation Trapped in the Earth's Magnetic Field, D. Reidel, Dordrecht, Holland, (1966).

[2] M. Schulz, Geomagnetically Trapped Radiation, Sp. Sci. Rev. 17, 481 (1975).

[3] W.N. Spjeldvik, Expected Charge States of Energetic Ions in the Magnetosphere, Sp. Sci. Rev. 23, 499 (1979).

[4] T.A. Fritz, W. N. Spjeldvik, Experimental Determination of Geomagnetically Trapped Heavy Ion Fluxes, International Association of Aeronomy and Geomagnetism, International Symposium, Edinburgh, Scotland, August, (1981).

[5] M. Walt, Introduction to Geomagnetically Trapped Radiation, Cambridge Univ. Press, New York, (1994).

[6] W.N. Spjeldvik, Consequences of the Duration of Solar Energetic Particle Associated Geomagnetic Storms on the Intensity of Geomagnetically Trapped Radiation, 311, in Space Physics Modeling, American Geophysical Union Monograph, $\mathrm{N}^{\circ}$ 44, Washington, DC, (1988).

[7] G.D. Reeves, (editor), Workshop on the Earth's Trapped Radiation environment, American Institute of Physics Conference Proceedings, No.383, New York, (1996).

[8] G.I. Pugacheva, A. A. Gusev, I. M. Martin, W. N. Spjeldvik, T. Kohno, The Temporal Evolution of $3-9$
$\mathrm{MeV} /$ nucleon He Flux Trapped in the Magnetosphere, Geophys. Res. Letters, 23, 2793 (1996).

[9] A.A. Gusev, T. Kohno, W. N. Spjeldvik, I. M. Martin, G. I. Pugacheva, and A. Turtelli, Jr., The 3-9 $\mathrm{MeV} /$ Nucleon He Ions Flux Dynamics in the Inner Magnetosphere Obtained with Ohzora Satellite, Adv. Space Res., 20-3, 385 (1997).

[10] A. Vacaresse, D. Boscher, S. Bourdarie, M. Blanc, and J.-A. Sauvaud, Modeling the High Energy Proton Belt, J. Geophys. Res., 104, 28601 (1999).

[11] T. Beutier, D. Boscher, and M. France, Salammbô, A Three-dimensional Simulation of the Proton Radiation Belt, J. Geophys. Res. 100, 17181 (1995).

[12] J.M. Cornwall, Radial Diffusion of Ionized Helium and Protons: A Probe for Magnetospheric Dynamics, J. Geophys. Res., 77, 1756 (1972).

[13] W.N. Spjeldvik, T. A. Fritz, Energetic Ionized Helium in the Quiet Time Radiation Belts: Theory and Comparison with Observation, J. Geophys. Res., 83, 654 (1978).

[14] W.N. Spjeldvik, T. A. Fritz, Observations of Energetic Helium Ions in the Earth's Radiation Belts During a Sequence of Magnetic Storms, J. Geophys. Res., 83, 2317 (1978).

[15] L.J. Lanzerotti, D. C. Webb, and C. W. Arthur, Geomagnetic Field Fluctuations at Synchronous Orbit, 2. Radial Diffusion, J. Geophys. Res., 83, 3866 (1978). 